\title{
Comparison of polarization and polarimetric optical tomography methods for recognition of tissue simulators inside breast phantom
}

\author{
Sławomir Miernicki, Piotr K. Sobotka, Andrzej W. Domański* \\ Faculty of Physics, Warsaw University of Technology, Koszykowa 75, 00-662 Warszawa,
}

Received December 22, 2011; accepted December 28, 2011; published December 31, 2011

\begin{abstract}
In the paper we present the results of tissue simulators recognition inside the breast phantom, based on polarization analysis of scattered light as well as polarimetric optical tomography method based on the Mueller-Stokes formalism. Blood, fat and muscle tissues were simulated by $10-\mathrm{mm}$ diameter probes with a red dye, $20 \%$ Intralipid $^{\mathrm{TM}}$ and water with polarizing foil respectively. All tissue simulators were placed inside a container $(120 \mathrm{~mm}$ in diameter) filled by $0.1 \%$ solution of Intralipid ${ }^{\mathrm{TM}}$. Different polarization states of a laser beam $(670 \mathrm{~nm}$, $35 \mathrm{~mW}$ ) were used for lighting the container (breast phantom). The results of the measurement show that polarization optical tomography enables much easier recognition of different kinds of tissue simulators. On the other hand, polarimetric optical tomography allows for better recognition of the angular placement of tissue simulators.
\end{abstract}

There are a few types of optical tomography based on analysis of scattered light in tissue. The most successful is optical coherence tomography (OCT) which allows to image the structure of a living eye or surface layers of skin tissues [1-3]. OCT requires a low scattering medium and it is not useful for imaging the breast structure with its high scattering tissues. Optical diffusion tomography (ODT) is based on analysis of photons diffusion in high scattering media and has been introduced for imaging the brain structure close to the skull [4-5]. ODT can be also applied for imaging of tumor inside the breast but with a relatively low accuracy [6].

There are other attempts for optical imaging of high scattering structures. Some of them are based on polarization analysis of scattered light, which depends on the structure of the medium, particularly when the Rayleigh scattering plays main role in comparison to the Mie scattering as it is the case of living tissue [7]. Since the Rayleigh scattering is the most sensitive to polarization perpendicular to the incoming beam, the right angle should be chosen between illuminating and detecting directions. Tomography imaging based on simple measurements of polarization states of scattered light is called polarization optical tomography. A more advanced method based on the Mueller-Stokes formalism is known as polarimetric optical tomography.

\footnotetext{
${ }^{*}$ E-mail: domanski@if.pw.edu.pl
}

The experimental set-up of both optical polarization and polarimetric imaging systems is shown in Fig. 1. A laser diode module operating at $670 \mathrm{~nm}(35 \mathrm{~mW})$ is used as a light source. The measurement system includes also a generator of polarization states consisting of a linear polarizer, a quarter-wave and half-wave plates. These optical components allow for modification of the input state of polarization.

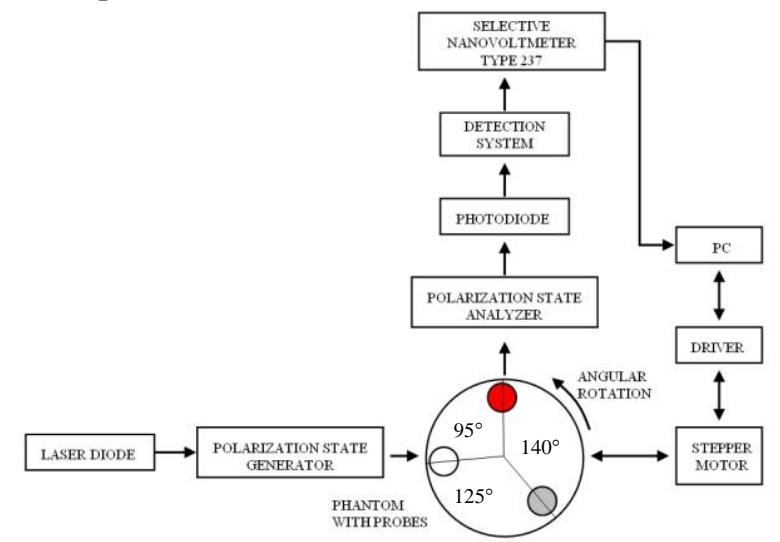

Fig. 1. Set-up of the optical polarization and polarimetric tomography system

As a scattering medium a solution of Intralipid ${ }^{\mathrm{TM}}$ in water $(0.1 \%)$ was used. It is a kind of the fat emulsion simulating optical properties of tissue [8]. Inside the container with an Intralipid ${ }^{\mathrm{TM}}$ solution $(120 \mathrm{~mm}$ diameter) we put three probes $(10 \mathrm{~mm}$ diameters) with high density Intralipid (20\%), water and polarizing foil as well as red dye simulating fat, muscle and blood, respectively (Fig. 2). It was shown that linearly, circularly and elliptically polarized light changes its polarization in a different way in birefringent media (e.g. muscle tissue) [9-10].The probes were placed with some angular asymmetry between them $\left(95^{\circ}, 125^{\circ}, 140^{\circ}\right)$, but at the same distance from the center of the container $(45 \mathrm{~mm})$. Light scattered in the direction of the photodiode passes through another series of polarizing optics which consists of a linear polarizer, quarter-wave plate and photodiode. The measurements are taken for angles from $0^{\circ}$ to $360^{\circ}$ according to the tomography angular scanning procedure. 

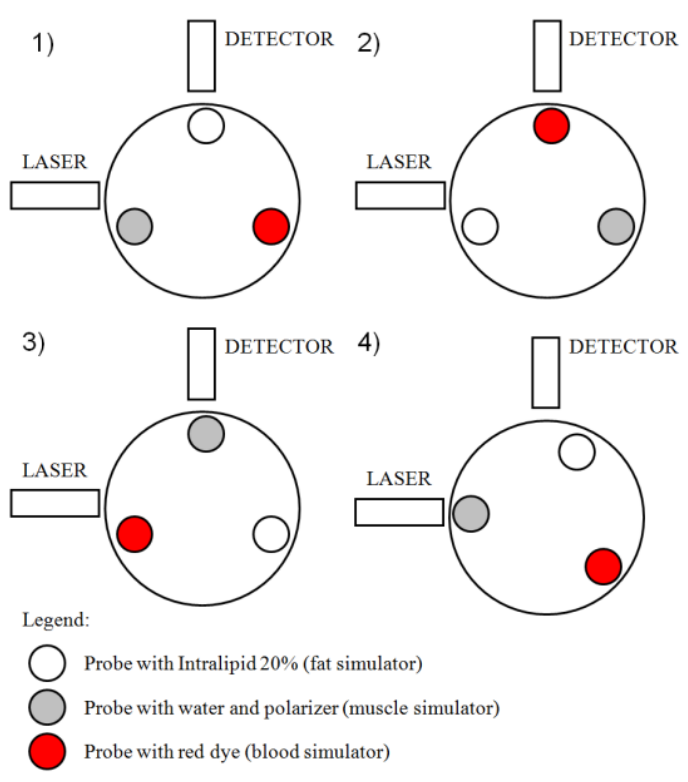

Fig. 2. Four the most interesting angular positions of breast phantom with tissue in experimental results.

An idea of polarization optical tomography is based on analysis of scattered light polarization for different polarization states of a laser beam illuminating the breast phantom. A linear polarized light beam is obtained by use horizontal $(\mathrm{H})$, vertical $(\mathrm{V})$ or $45^{\circ}(\mathrm{P})$ azimuths of the polarizer. A circular right-handed $(\mathrm{R})$ polarized light beam is obtained by using a quarter-wave plate. The scattered light is analyzed by the use of horizontal $(\mathrm{H})$, vertical $(\mathrm{V})$, and $45^{\circ}(\mathrm{P})$-oriented analyzer as well as a quarter-wave plate $(\mathrm{R})$. Each angular step requires sixteen intensity measurements according to the combinations of the states of polarization represented by the matrix:

$$
\left[\begin{array}{lllll}
H H & H V & H P & H R \\
V H & V V & V P & V R \\
P H & P V & P P & P R \\
R H & R V & R P & R R
\end{array}\right]
$$

where the first letter of an element means state of polarization of an incoming beam and the second letter means the polarization element used in the detecting channel. Angular scanning with a step of $1^{\circ}$ gives $16 \times 360$ measurements results shown in Fig. 3.

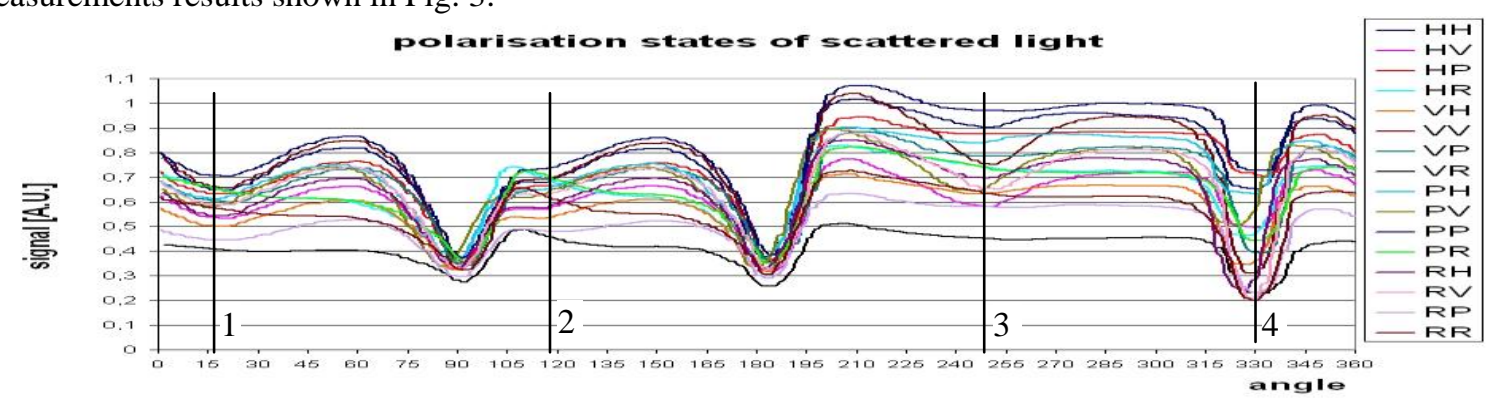

Fig. 3 . Experimental results of intensity measurements during angular scanning for sixteen polarization combination; (1), (2), (3), (4) mean angular positions of phantom shown in Fig 2. shown in Fig. 4.
Polarimetric optical tomography is based on analysis of Mueller matrix [M] elements. The matrix equation describing polarization changes introduced by a scattering medium is given by:

$$
\left[\mathrm{S}^{\mathrm{OUT}}\right]=[\mathrm{M}]\left[\mathrm{S}^{\mathrm{IN}}\right]
$$

where the Stokes vector of an incoming beam may have a simple form:

-for horizontally oriented linear polarization:

$\left[\mathrm{S}_{\mathrm{H}}^{\mathrm{IN}}\right]=[\mathrm{H}, \mathrm{H}, 0,0]$, vertical oriented: $\left[\mathrm{S}_{\mathrm{V}}{ }^{\mathrm{IN}}\right]=[\mathrm{V},-\mathrm{V}, 0,0]$, $45^{\circ}$ oriented: $\left[\mathrm{S}_{\mathrm{P}}{ }^{\mathrm{IN}}\right]=[\mathrm{P}, 0, \mathrm{P}, 0]$,

-for right-handed circular polarization: $\left[\mathrm{S}_{\mathrm{R}}{ }^{\mathrm{IN}}\right]=[\mathrm{R}, 0,0, \mathrm{R}]$.

The Stokes vector of scattered light is calculated on the basis of intensity measurements according to formula:

$$
\left[\begin{array}{l}
S_{0} \text { out } \\
S_{1}{ }^{\text {out }} \\
S_{2} \text { out } \\
S_{3}{ }^{\text {out }}
\end{array}\right]=\left[\begin{array}{c}
H+V \\
H-V \\
2 P-H-V \\
2 R-H-V
\end{array}\right],
$$

where $\mathrm{H}, \mathrm{V}, \mathrm{P}, \mathrm{R}$ mean the intensities of scattered light measured with the help of horizontal $(\mathrm{H})$, vertical $(\mathrm{V}), 45^{\circ}$ azimuth $(\mathrm{P})$ of an analyzer and a quarter wave plate $(\mathrm{R})$.

It may be proved [11] that the solution of the matrix equation [Eq. (2)] with the help of 16 polarization measurements shown in the polarization matrix [Eq. (1)] gives all 16 Mueller matrix elements:

$$
[M]=\left[\begin{array}{llll}
m_{00} & m_{01} & m_{02} & m_{03} \\
m_{10} & m_{11} & m_{12} & m_{13} \\
m_{20} & m_{21} & m_{22} & m_{23} \\
m_{30} & m_{31} & m_{32} & m_{33}
\end{array}\right]=
$$

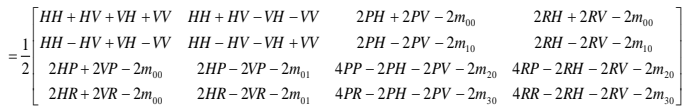

The results of the Mueller matrix element calculations are 


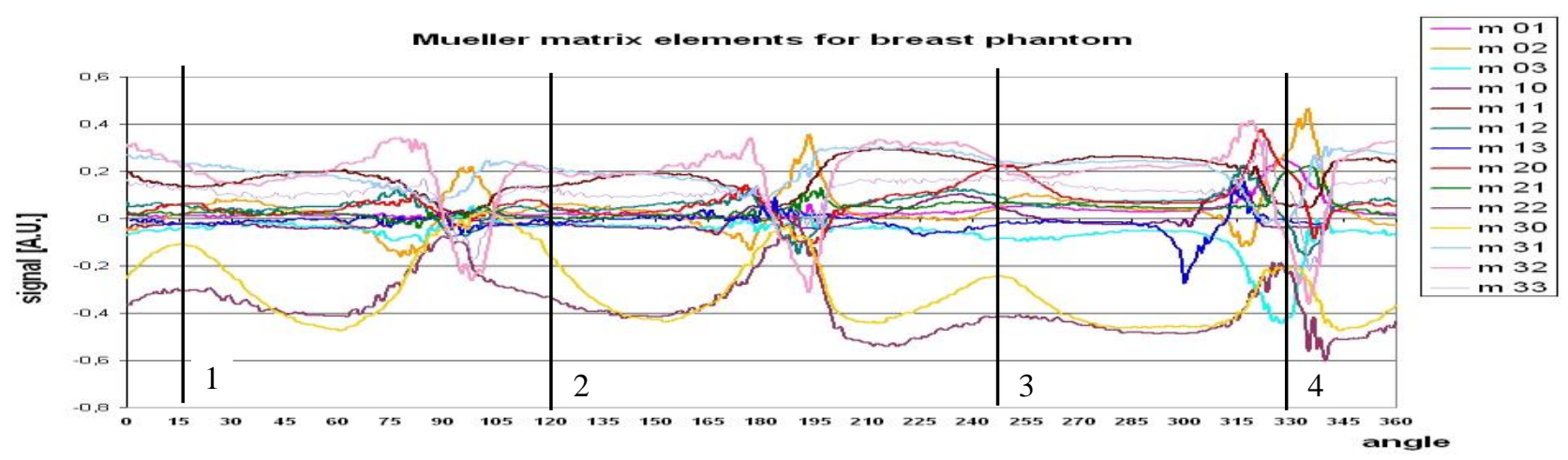

Fig. 4. Results of Mueller matrix elements calculations; (1), (2), (3), (4) mean angular position of tissue simulators shown in Fig. 3.

We choose four angular positions of the phantom in order to compare polarization and polarimetric optical tomography methods for the recognition of tissue simulators (Fig. 2). Each position is marked by a vertical line in both sets of the results shown in Figs. 3 and 4.

Based on the results of polarization measurements (Fig.3), we can conclude that only the probe with a blood simulator close to the detector (angular position (2) in Fig. 2) gives no local minimum seen in Fig. 3, although two other probes have such minima (angular position (1) for a fat simulator and position (3) for a muscle simulator in Fig. 2) and their number is different in both cases. It can be also observed in Fig. 3 that only the probe with a muscle simulator taking position close to the lighting beam (angular position (4) in Fig. 2) gives not so deep minima for some measurements as two other tissue simulators.

Additional calculation of polarization measurement leading to the Mueller matrix elements gives a more complicated view on analysis as can be seen in Fig. 4. Nevertheless, $\mathrm{m}_{02}$ and $\mathrm{m}_{32}$ elements allow to show the exact placement of probes (Fig. 5). In addition, the polarimetric tomography method also allows to recognize tissue simulators inside the breast phantom and gives us a more advanced tool for analysis as well.

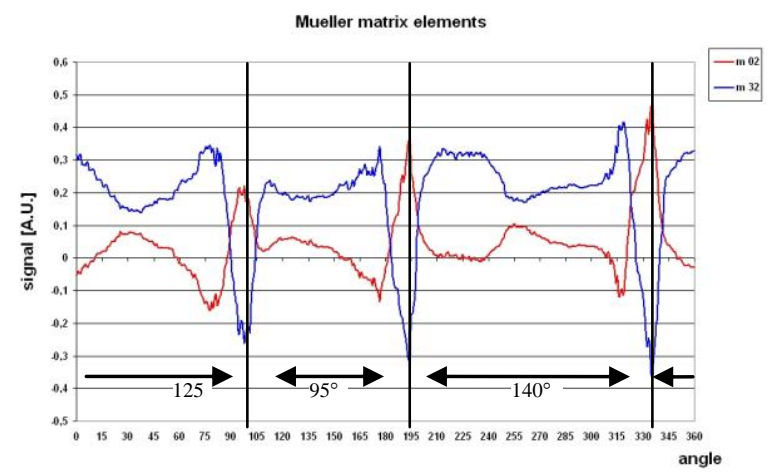

Fig. 5 Two Mueller matrix elements $\left(\mathrm{m}_{02}\right.$ and $\left.\mathrm{m}_{32}\right)$

showing exact placement of probes with tissue simulators.
The work leading to the paper was supported by the Polish Ministry of Science and Education under the grand No N N518 385237 and the European Social Fund implemented under the Human Capital Operational Programme (POKL), project: "Preparation and Realization of Medical Physics Specialty".

\section{References}

[1] D. Huang, E.A. Swanson, C.P. Lin, J.S. Schuman, W.G. Stinson, W. Chang, M.R. Hee, T. Flotte, K. Gregory, C.A. Puliafito, J.G. Fujimoto, Science 254, 1178(1991).

[2] A.F. Fercher, W. Drexler, C.K. Hitzenberger, T. Lasser, Rep. Prog. Phys. 66, 239 (2003).

[3] T. Bajraszewski, I. Gorczyńska, A. Szkulmowska, M. Szkulmowski, P. Targowski, A. Kowalczyk, Proc. SPIE 5959, 65 (2005).

[4] A.H. Hielscher, A.D. Klose, K.M. Hanson, IEEE Trans. Med. Imaging 18(3),262(1999).

[5] T. Tarvainen, M.Vauhkonen, V. Kolehmainen, J.P. Kaipio, S.R. Arridge, PIERS Online 4(6),655 (2008).

[6] T. Nielsen, B. Brendel, R. Ziegler, M. Beek, F Uhlemann, C. Bontus, T. Koehler, App. Optics 48(10),D1 (2009).

[7] A.W. Domański, M. Rytel, T.R. Wolinski, Proc. SPIE 5959, 1 (2005).

[8] R. Michels, F. Foschum, A. Kienle, Opt. Exp.16(8),5907 (2008).

[9] Ł. Michalik, M. Redek, P. Makowski, A.W. Domański, Phot. Lett. Poland 1(4), 184 (2009).

[10] A.W. Domański, M. Redek, D. Budaszewski, Phot. Lett. Poland, 1(2), 67 (2009).

[11] G. Yao, L.V. Wang, Opt. Lett. 24(8), 537 (1999). 